\title{
Study Protocol for Transcatheter Aortic Valve Replacement for a Degenerated Aortic Bioprosthesis in a Japanese Cohort
}

\author{
Kizuku Yamashita, MD; Satsuki Fukushima, MD, PhD; Yusuke Shimahara, MD; \\ Takuma Yamasaki, MD; Yorihiko Matsumoto, MD; Naonori Kawamoto, MD; \\ Naoki Tadokoro, MD; Takashi Kakuta, MD; Yasuhiro Hamatani, MD; \\ Atsushi Okada, MD; Hiroyuki Takahama, MD, PhD; Makoto Amaki, MD, PhD; \\ Takuya Hasegawa, MD, PhD; Hideaki Kanzaki, MD, PhD; Chisato Izumi, MD, PhD; \\ Satoshi Yasuda, MD, PhD; Junjiro Kobayashi, MD, PhD; Tomoyuki Fujita, MD, PhD
}

\begin{abstract}
Background: The valve-in-valve (VIV) procedure is being increasingly performed in high-risk patients with a degenerated bioprosthesis in an aortic position in Western countries. The early safety and efficacy of the VIV procedure, however, remain unclear in Japanese patients with a small aortic annulus. We present the protocol for a study designed to evaluate the early safety and efficacy of the VIV procedure in the aortic position in Japanese patients.

Methods and Results: The prospective, single-center, non-comparative, clinical study of the VIV procedure for the aortic position (AORTIC VIV study) commenced in August 2016 and will end in March 2020. Patients will be monitored for $\geq 1$ month after the VIV procedure. The targeted number of patients is 11. Eligible patients are those who have undergone transcatheter aortic valve replacement for a surgical valve (including stented or stentless bioprosthetic valves), or for a transcatheter heart valve. The VIV procedure is performed in high-operative-risk patients with substantial prosthetic valve stenosis, and regurgitation and heart failure resistant to medical treatment (unless the patient meets an exclusion criterion). The safety and efficacy of the VIV procedure will be evaluated in accordance with the Valve Academic Research Consortium-2 initiative.
\end{abstract}

Conclusions: The AORTIC VIV study will clarify the early safety and efficacy of the VIV procedure in Japanese patients.

Key Words: Transcatheter aortic valve replacement; TAV-in-SAV; TAV-in-TAV; Valve-in-valve

$\mathbf{T}$ ranscatheter aortic valve replacement (TAVR) has become an established procedure in recent years. Moreover, for high-risk patients who have previously undergone aortic valve replacement (AVR), TAVR in the failed surgical bioprosthesis (valve-in-valve procedure) has been reported since 2007. ${ }^{1}$ The safety and efficacy of the valve-in-valve procedure have been recognized, and this procedure is being performed worldwide., ${ }^{2,3}$ Recent guidelines recommend the valve-in-valve procedure in the aortic position for patients considered to be at high or prohibited risk for reoperation., ${ }^{4,5}$ A major concern regarding the valve-in-valve procedure, however, is the presence of a previously implanted small surgical valve $(\leq 21 \mathrm{~mm})$, because small surgical valve is an independent risk factor for 1-year mortality after the valve-in-valve procedure. ${ }^{3}$ This is particularly concerning in Japan, where a $19-\mathrm{mm}$ or $21-\mathrm{mm}$ bioprosthesis is used in $57-75 \%$ of AVR. ${ }^{68}$ To explore the clinical outcomes of the valve-in-valve procedure in Japanese patients, we established a clinical study entitled "A single institution, exploratory, safety evaluation study of AORTIC Valve-In-Valve transcatheter aortic valve implantation for degenerated bioprosthetic heart valve" (AORTIC VIV study). Consequently, we briefly reported the operative outcomes of the valve-in-valve procedure in the aortic position in 3 patients with failed $19-\mathrm{mm}$ stented bioprostheses. ${ }^{9}$ The outcomes of the valve-in-valve procedure (consisting of the insertion of a transcatheter aortic valve in a surgical aortic stented valve of other sizes or in a stentless valve, or the insertion of a transcatheter aortic valve in a transcatheter aortic valve), in the Japanese population, however, remain unclear. Herein, we describe the protocol of the AORTIC VIV study. The findings of the ongoing AORTIC VIV study will aid in the achievement of better outcomes of the valve-in-valve procedure in the

Received December 10, 2018; accepted December 11, 2018; J-STAGE Advance Publication released online January 19, 2019 Time for primary review: 1 day

Department of Cardiac Surgery (K.Y., S.F., Y.S., T.Y., Y.M., N.K., N.T., T.K., J.K., T.F.), Department of Cardiovascular Medicine (Y.H., A.O., H.T., M.A., T.H., H.K., C.I., S.Y.), National Cerebral and Cardiovascular Center, Suita, Japan; William Harvey Research Institute, Barts and The London School of Medicine and Dentistry, Queen Mary University of London, London (K.Y.), UK

Mailing address: Tomoyuki Fujita, MD, PhD, Director, Department of Cardiac Surgery, National Cerebral and Cardiovascular Center, 5-7-1 Fujishirodai, Suita 565-8565, Japan. E-mail: tomofujita@nifty.com

ISSN-2434-0790 All rights are reserved to the Japanese Circulation Society. For permissions, please e-mail: cr@j-circ.or.jp 
Japanese population.

\section{Methods}

\section{Study Design}

The AORTIC VIV study is a prospective, non-comparative, non-randomized, interventional cohort study that aims to evaluate the off-label use of a commercially available transcatheter heart valve (THV) in high-risk patients with a degenerated bioprosthesis in the aortic position in Japan. The study has been approved by the National Cerebral and Cardiovascular Center Institutional Review Board (approval number: M28-046-5). We aim to include 11 patients with a degenerative bioprosthesis in the aortic position. The AORTIC VIV study data collection began in August 2016, and is scheduled to end in March 2020. All included patients will provide written informed consent for the procedure and for the use of their data for diagnostic and research purposes. The study is registered in the University Hospital Medial Information Network Clinical Trials Registry (identifier UMIN000026450).

\section{Patient Selection, Data Storage, and Sample Size}

Patients are screened to ensure that they meet all of the following inclusion criteria: (1) age $\geq 20$ years; (2) substantial deterioration of the implanted prosthetic valve, including degenerated THV, stenosis, regurgitation, or both; $;^{\mathbf{1 0} 11}$ (3) heart failure resistant to medical treatment; (4) a consensus among the institutional heart team (consisting of cardiologists and cardiac surgeons) that the patient has a high or prohibited operative risk for redo open heart surgery; and (5) provision of written informed consent for this procedure (Table 1). High operative risk was defined as an expected mortality risk $>8.0 \%$ as estimated on Society of Thoracic Surgeons score, ${ }^{12}$ and/or the presence of the following risk factors that were considered to render the case inoperable (at the surgeons' discretion): porcelain aorta, mobile atherosclerosis at the aorta, history of mediastinal radiotherapy or mediastinitis, age $>75$ years with a left ventricular (LV) ejection fraction (EF) $<40 \%$, previous coronary artery bypass grafting, history of $\geq 2$ cardiac surgeries, or liver cirrhosis.

The exclusion criteria are: (1) allergy to any type of metal; (2) allergy to contrast media; (3) thrombus in the LV or atrium on transesophageal echocardiography (TEE); (4) shock; (5) infection or degeneration of the bioprosthesis due to active infective endocarditis; (6) hemodialysis; (7) mechanical heart valve in the aortic position; (8) pregnancy,
Table 1. Inclusion Criteria

- Patient age $\geq 20$ years

- Substantial deterioration of an implanted prosthetic valve

- Heart failure resistant to medical treatment

- High or prohibited operative risk

- Provision of written informed consent

nursing, possibility of pregnancy, or desire to become pregnant during the study period; (9) participation in another investigational study or intention to participate in another investigational study during the study period; and (10) any other reason that the investigators consider renders the patient inappropriate for study participation (Table 2).

Preoperative screening of each patient is performed. The anatomical structures in the aortic position are evaluated using electrocardiogram-gated multidetector computed tomography (ECG-gated MDCT; Somatom Definition Flash, Siemens Healthcare, Erlangen, Germany) to confirm the anatomy of the aortic complex, prosthetic valve, and access site. After MDCT, the graphical data are analyzed and reconstructed using an imaging workstation (Ziostation2; Ziosoft, Tokyo, Japan). The severity of THV degeneration is assessed via transthoracic echocardiography (TTE) and TEE. Coronary angiography is performed to detect coronary artery lesions. The flowchart of the study is shown in Figure 1.

After confirmation of eligibility, patient data are registered and collected in a National Cerebral and Cardiovascular Center electronic data capture system. ${ }^{13}$ The study data will be confidentially stored for 10 years after the publication of the AORTIC VIV study, and will not be provided to other institutes.

The estimated number of patients who will undergo redo surgical AVR at the National Cerebral and Cardiovascular Center annually is approximately $4-5$. Therefore, it is estimated that 11 patients will be eligible for inclusion in the AORTIC VIV study.

\section{Study Endpoints and Follow-up}

The primary endpoints consist of the intraoperative safety and intraoperative efficacy (device success) based on the Valve Academic Research Consortium (VARC)-2 initiative. ${ }^{10}$ Intraoperative safety will be assessed based on the rates of all-cause mortality, all types of stroke, life-threatening bleeding including perforation of the heart and

Table 2. Exclusion Criteria
- Allergy to any type of metal
- Allergy to contrast media
- Thrombus in the LV or atrium detected on TEE
- Shock
- Infection or degeneration of the bioprosthesis due to active infective endocarditis
- Hemodialysis
- Mechanical heart valve in the aortic position
- Pregnancy, nursing, or possibility of pregnancy
- Participation in another investigational study or intention to participate in another investigational study during the study period
- Any other reason that the investigators consider renders the patient inappropriate for study participation

LV, left ventricle; TEE, transesophageal echocardiography. 


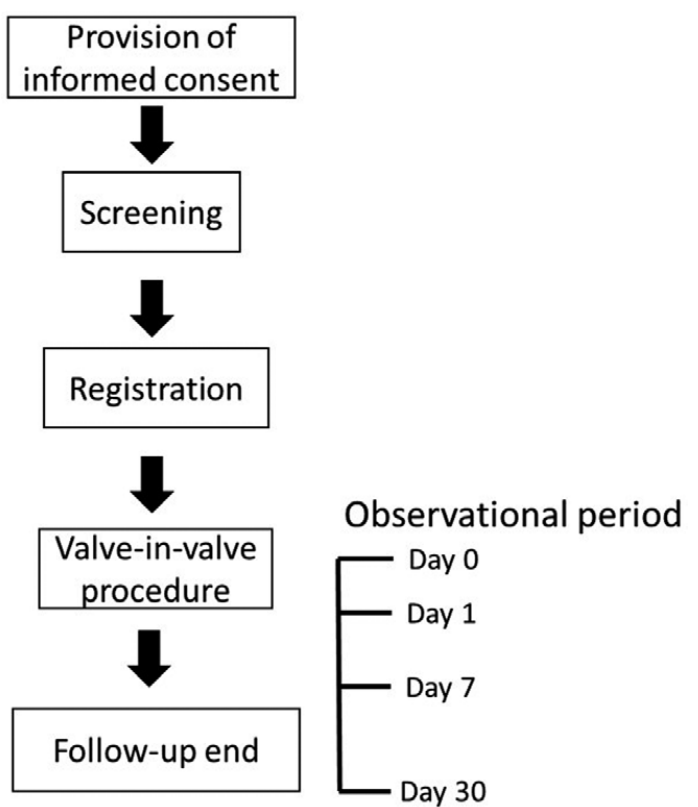

Figure 1. Flowchart of the AORTIC VIV study. Patients will be followed up for as long as possible after the observational period. VIV, valve-in-valve. cardiac tamponade, cardiogenic shock, and prosthetic valve failure soon after the procedure. Device success is defined as absence of procedural mortality, correct positioning in a single valve-in-valve procedure, and attainment of the intended performance of the prosthetic heart valve (no severe prosthesis-patient mismatch $\left[>0.65 \mathrm{~cm}^{2} / \mathrm{m}^{2}\right]$, mean aortic valve gradient $<20 \mathrm{mmHg}$ or peak aortic jet velocity $<3 \mathrm{~m} / \mathrm{s}$, and no moderate or severe prosthetic valve regurgitation). The secondary endpoints are the early safety and early therapeutic efficacy within 30 days after the procedure based on the VARC-2 initiative. Early safety will be assessed based on the rates of all-cause mortality, all types of stroke, life-threatening bleeding including perforation of the heart and cardiac tamponade, cardiogenic shock, requirement of intervention for coronary artery disease, and prosthetic valve failure within 30 days after the procedure. Early therapeutic efficacy will be assessed in accordance with prosthetic valve function, New York Heart Association (NYHA) classification, plasma brain natriuretic peptide (BNP) concentration, walking distance in $6 \mathrm{~min}$ (6-min walk test), LV end-diastolic diameter/LV endsystolic diameter, and LVEF on TTE, pulmonary artery wedge pressure (PAWP), and cardiac index. Prosthetic valve function (including peak aortic jet velocity, aortic peak/mean pressure gradient, effective orifice area, and regurgitation) will be assessed at baseline, and at 7 and 30 days postoperatively. NYHA functional classification, plasma BNP concentration, LV end-diastolic diameter, LV end-systolic diameter, and LVEF will be measured at baseline, and at 7 and 30 days postoperatively. The 6-min walk test will be conducted at baseline and at 7 days postoperatively. The PAWP and cardiac index will be assessed

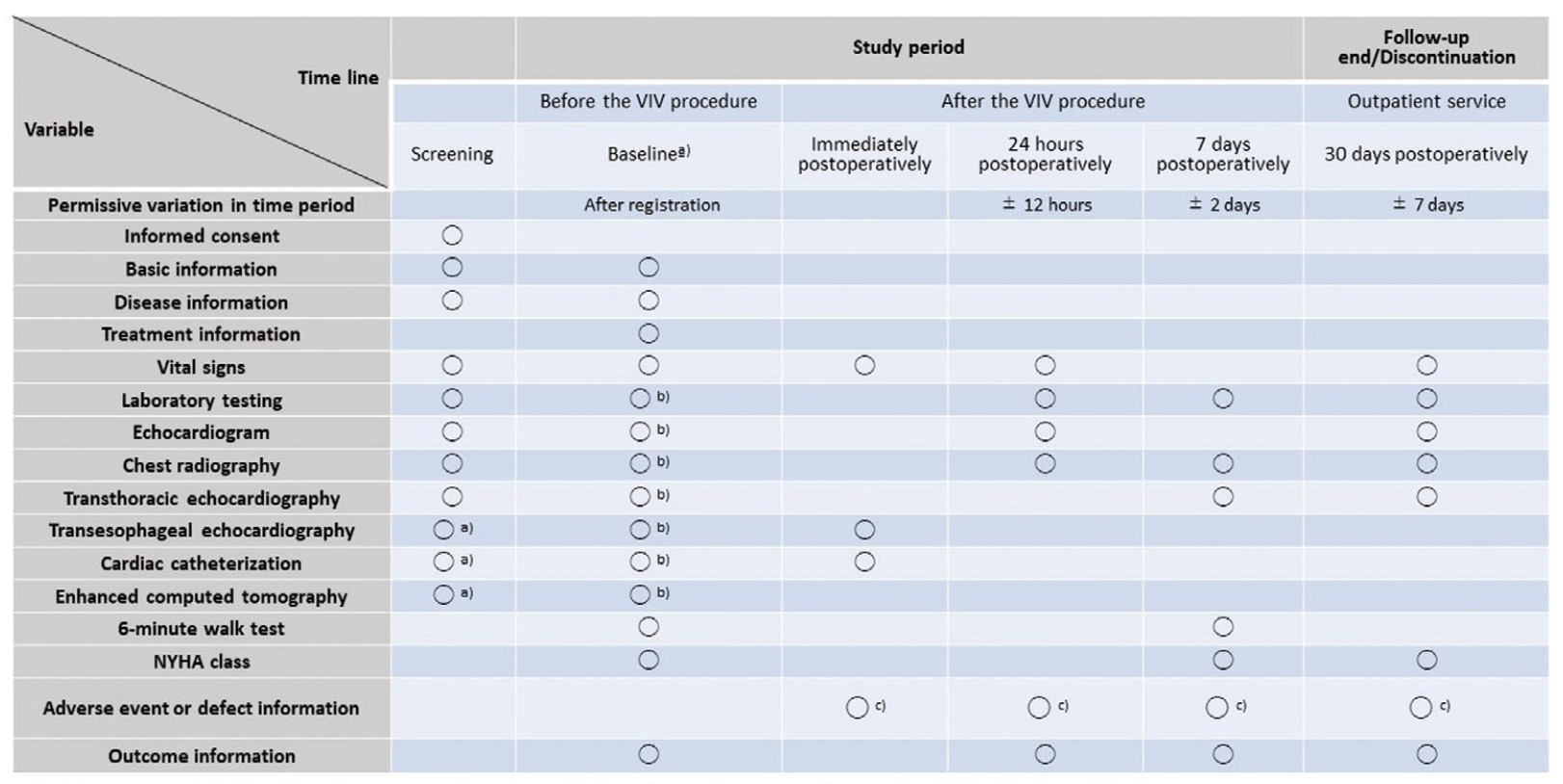

a) It is acceptable to skip these examinations if they have been performed within the last 90 days.

b) Examinations at baseline may be skipped if they have been performed during screening.

c) Adverse event or defect information will be evaluated as soon as possible.

Figure 2. Timeline of the preoperative and postoperative examinations in the AORTIC VIV study. NYHA, New York Heart Association; VIV, valve-in-valve. 
at baseline and immediately postoperatively. All patients will be followed up for $\geq 30$ days postoperatively, and then for as long as possible. The timeline of examinations is shown in Figure 2.

\section{Valve-in-Valve Procedure and Device Selection}

Each patient is placed in the supine position under general anesthesia with endotracheal intubation. The selected THV is implanted with intraoperative fluoroscopic guidance and TEE. A temporary pacemaker (Harmac Medical Products, Buffalo, NY, USA) is inserted via the right internal jugular vein to control the intraoperative pacing during the deployment and to preclude bradycardia or atrioventricular block. The valve-in-valve procedure is performed via the standard method. ${ }^{14} \mathrm{~A}$ balloon-expandable THV is deployed under rapid pacing of $150-220$ beats $/ \mathrm{min} .{ }^{15} \mathrm{~A}$ final angiogram is performed to confirm the deployed position and to check for paravalvular or transvalvular leakage around the deployed THV.

To minimize invasiveness, the transfemoral approach is chosen as the first access. The SAPIEN XT (Edwards Lifesciences, Irvine, CA, USA) balloon-expandable THV, and the CoreValve Evolut R (Medtronic, Minneapolis, MN, USA) will be used in this study. The selection of the THV and size will depend on the anatomical eligibility of each THV and the consensus decision of the institutional heart team.

\section{Event Assessment and Safety Monitoring}

All clinical events relevant to this procedure will be reported by the investigators as soon as possible, and the events will be recorded in the electronic data capture system using a regulated form. Experts will individually review the event, assess the cause and the clinical data, and follow up the affected patient.

The safety monitoring committee evaluates clinical events and any other relevant adverse events. All clinical data and events will be assessed by the safety monitoring committee during the study period.

\section{Statistical Analysis}

All preoperative and postoperative demographic and clinical characteristics will be expressed as medians (IQRs) for continuous variables, and as n (\%) for categorical variables. The Wilcoxon rank-sum test will be conducted for continuous variables, and the chi-squared test will be used for categorical variables. The chi-squared test or Fisher's exact test will be used to compare categorical variables. Two-sided $\mathrm{P}<0.05$ will be considered to indicate statistical significance.

\section{Discussion}

There are several issues related to the valve-in-valve procedure. The most catastrophic complications are coronary obstruction and valve malpositioning, especially in stentless bioprostheses. ${ }^{16-18}$ To preclude these complications in the present study, the details of the aortic complex including coronary height and size of the sinus of Valsalva will be collected in all cases, and the percutaneous coronary intervention team and extracorporeal membrane oxygenation will be available at all times for emergency intervention. To treat the migration of the implanted THV promptly, the heart team members were trained via the protocol of the AORTIC VIV study before starting to perform the valve- in-valve procedure. The AORTIC VIV study findings will be useful for the performance of the valve-in-valve procedure in the Japanese population. In the TAVR era, the durability of the THV remains unknown. It is important to continue obtaining the follow-up data for as long as possible after the study ends.

\section{Study Limitations}

This is a prospective, single-center, non-comparative study with a small number of patients. The decision-making regarding the THV selection and access route is dependent on the heart team.

\section{Conclusions}

The AORTIC VIV study will clarify the early feasibility, safety, and efficacy of the aortic valve-in-valve procedure in selected high-risk Japanese patients with degenerated aortic bioprostheses. This information will help to preclude complications related to the valve-in-valve procedure in Japanese patients with a small aortic annulus.

\section{Acknowledgments}

This work is supported by the "Investigation for efficacy and safety of minimally invasive valve surgery (27-3-1) for Cardiovascular Diseases of National Cerebral and Cardiovascular Center". We thank Kelly Zammit, BVSc, from Edanz Editing (www.edanzediting.com/ac), for editing a draft of this manuscript. Roles and responsibilities of this study are listed in Supplementary File.

\section{Disclosures}

T.F. is an advisor for Medtronic. Devices were provided without compensation by Edwards Lifesciences and Medtronic.

\section{References}

1. Wenaweser P, Buellesfeld L, Gerckens U, Grube E. Percutaneous aortic valve replacement for severe aortic regurgitation in degenerated bioprosthesis: The first valve in valve procedure using the Corevalve Revalving system. Catheter Cardiovasc Interv 2007; 70: 760-764.

2. Dvir D, Webb J, Brecker S, Bleiziffer S, Hildick-Smith D, Colombo A, et al. Transcatheter aortic valve replacement for degenerative bioprosthetic surgical valves: Results from the global valve-in-valve registry. Circulation 2012; 126: 2335-2344.

3. Dvir D, Webb JG, Bleiziffer S, Pasic M, Waksman R, Kodali S, et al. Transcatheter aortic valve implantation in failed bioprosthetic surgical valves. JAMA 2014; 312: 162-170.

4. Nishimura RA, Otto CM, Bonow RO, Carabello BA, Erwin JP 3rd, Fleisher LA, et al. 2017 AHA/ACC Focused Update of the 2014 AHA/ACC Guideline for the Management of Patients With Valvular Heart Disease: A Report of the American College of Cardiology/American Heart Association Task Force on Clinical Practice Guidelines. Circulation 2017; 135: e1159-e1195.

5. Baumgartner H, Falk V, Bax JJ, De Bonis M, Hamm C, Holm PJ, et al. 2017 ESC/EACTS guidelines for the management of valvular heart disease. Eur Heart J 2017; 38: 2739-2791.

6. Matsumoto Y, Fujita T, Hata H, Shimahara Y, Sato S, Kobayashi J. Hemodynamic performance and durability of mosaic bioprostheses for aortic valve replacement, up to 13 years. Circ J 2015; 79: 1044-1051.

7. Kume Y, Fujita T, Fukushima S, Hata H, Shimahara Y, Matsumoto Y, et al. Reducing prosthesis-patient mismatch with Edwards magna prosthesis for aortic valve replacement. Circ J 2017; 81: $468-475$.

8. Minakata K, Tanaka S, Okawa Y, Shimamoto M, Kaneko T, Takahara Y. Long-term outcome of the Carpentier-Edwards pericardial valve in the aortic position in Japanese patients. Circ J 2014; 78: 882-889.

9. Yamashita K, Fujita T, Fukushima S, Shimahara Y, Kume Y, Matsumoto Y, et al. Transcatheter aortic valve implantation for degenerated 19-mm aortic bioprosthetic valve. Circ J 2017; 82: $289-292$. 
10. Kappetein AP, Head SJ, Généreux P, Piazza N, van Mieghem $\mathrm{NM}$, Blackstone $\mathrm{EH}$, et al. Updated standardized endpoint definitions for transcatheter aortic valve implantation: The Valve Academic Research Consortium-2 consensus document. $J$ Am Coll Cardiol 2012; 60: 1438-1454.

11. Zoghbi WA, Chambers JB, Dumesnil JG, Foster E, Gottdiener JS, Grayburn PA, et al. Recommendations for evaluation of prosthetic valves with echocardiography and Doppler ultrasound: A report from the American Society of Echocardiography's Guidelines and Standards Committee and the Task Force on Prosthetic Valves, developed in conjunction with the American College of Cardiology Cardiovascular Imaging Committee, Cardiac Imaging Committee of the American Heart Association, the European Association of Echocardiography, a registered branch of the European Society of Cardiology, the Japanese Society of Echocardiography and the Canadian Society of Echocardiography, endorsed by the American College of Cardiology Foundation, American Heart Association, European Association of Echocardiography, a registered branch of the European Society of Cardiology, the Japanese Society of Echocardiography, and Canadian Society of Echocardiography. $\mathrm{J} \mathrm{Am}$ Soc Echocardiogr 2009; 22: 975-1014.

12. Shroyer AL, Coombs LP, Peterson ED, Eiken MC, DeLong ER, Chen A, et al. The Society of Thoracic Surgeons: 30-day operative mortality and morbidity risk models. Ann Thorac Surg 2003; 75: $1856-1864$.

13. Harris PA, Taylor R, Thielke R, Payne J, Gonzalez N, Conde JG. Research electronic data capture (REDCap): A metadatadriven methodology and workflow process for providing trans- lational research informatics support. J Biomed Inform 2009; 42: $377-381$.

14. Gurvitch R, Cheung A, Ye J, Wood DA, Willson AB, Toggweiler $\mathrm{S}$, et al. Transcatheter valve-in-valve implantation for failed surgical bioprosthetic valves. J Am Coll Cardiol 2011; 58: 21962209.

15. Webb JG, Pasupati S, Achtem L, Thompson CR. Rapid pacing to facilitate transcatheter prosthetic heart valve implantation. Catheter Cardiovasc Interv 2006; 68: 199-204.

16. Dvir D, Leipsic J, Blanke P, Ribeiro HB, Kornowski R, Pichard $\mathrm{A}$, et al. Coronary obstruction in transcatheter aortic valve-invalve implantation: Preprocedural evaluation, device selection, protection, and treatment. Circ Cardiovasc Interv 2015; 8: pii: $\mathrm{e} 002079$.

17. Ribeiro HB, Rodés-Cabau J, Blanke P, Leipsic J, Kwan Park J, Bapat V. Incidence, predictors, and clinical outcomes of coronary obstruction following transcatheter aortic valve replacement for degenerative bioprosthetic surgical valves: Insights from the VIVID registry. Eur Heart $J$ 2018; 39: 687-695.

18. Huczek Z, Grodecki K, Scisło P, Wilczek K, Jagielak D, Fil W, et al. Transcatheter aortic valve-in-valve implantation in failed stentless bioprostheses. J Interv Cardiol 2018; 31: 861-869.

\section{Supplementary Files}

Please find supplementary file(s);

http://dx.doi.org/10.1253/circrep.CR-18-0023 